\section{X-Ray Analysis in the Low Vacuum SEM}

(Part 1 of 3 )

Don Chernoff, Small World

Low vacuum and environmental SEMs have become commonplace in many labs for many applications. With the rise in popularity of these instruments comes the need to understand their capabilities and limitations. An article I wrote in this publication in August 1997 summarized the issue of beam scatter in the low vacuum SEM. In this first of a series of three articles, I will go into more detail and give more examples on the phenomena of beam scatter in the low vacuum SEM.

The problem of beam scatter in the low vacuum SEM chamber is well known. Electrons from the primary beam interact with gas molecules in the SEM chamber, scatter, and hit the sample surface some distance away from the primary beam. The result is a "skirt" of electrons much wider than the primary beam. The amount of scatter depends on four variables: chamber pressure, working distance, accelerating voltage, and gas type. I will discuss the first three in this paper. The effect of gas type will be addressed in the next issue.

Image acquisition, whether by backscattered or secondary electrons, is largely unaffected by the skirt of electrons. Under typical low vacuum conditions most of the image signal is generated by the primary beam and the effect of the electron skirt can be ignored. Unfortunately this is not the case with $\mathrm{X}$-ray analysis. The electron skirt will give rise to $\mathrm{X}$-rays being generated some distance away from the primary beam. The extent of the problem depends on the size of the feature being analyzed and the diameter of the electron skirt. If the area being analyzed stays within the electron skirt, the effect of beam scatter on X-ray analysis can also be ignored. However, under typical low vacuum conditions as shown in the following examples, it does not take much for the electron skirt to be larger than the analysis area. The rest of this discussion will examine how the electron skit size depends on chamber pressure, working distance, and accelerating voltage.

The three sets of models clearly show that it is possible to keep beam scatter and the resulting electron skirt to a minimum by reducing the chamber pressure, reducing the working distance, and increasing the accelerating voltage. Conversely, the size of the electron skirt can become quite large, up to several millimeters, if the chamber pressure is too high, the working distance too long, or the accelerating voltage too low. It is up to the analyst to select the best combination of these conditions for the analysis being performed.

\section{Conclusion}

Beam scatter in the low vacuum or environmental SEM can generate $X$-ray signals far away from the area being analyzed. Four variables control the amount of beam scatter and size of the electron skirt: chamber pressure, working distance, accelerating voltage, and gas type. The analyst should be aware of the extent of the problem and adjust the instrument to keep the electron skirt to a minimum.

Part 2 in this series will discuss the effect of gas type on beam scatter.

The models shown in this article were generated by the software program "Electron Flight Simulator" version $\mathrm{E}$.

\section{References}

Small World: Electron Flight Simulator, version E (1997) http://members.aol.com/ smworld 100/index.htm dchernoff@aol.com

(703) 849-1492

Philips Electron Optics and Robert Johnson Associates:

Environmental Scanning Electron Microscopy, An Introduction to ESEM (1996)

Joy, David: Environmental SEM Monte Carlo Simulation (1997)

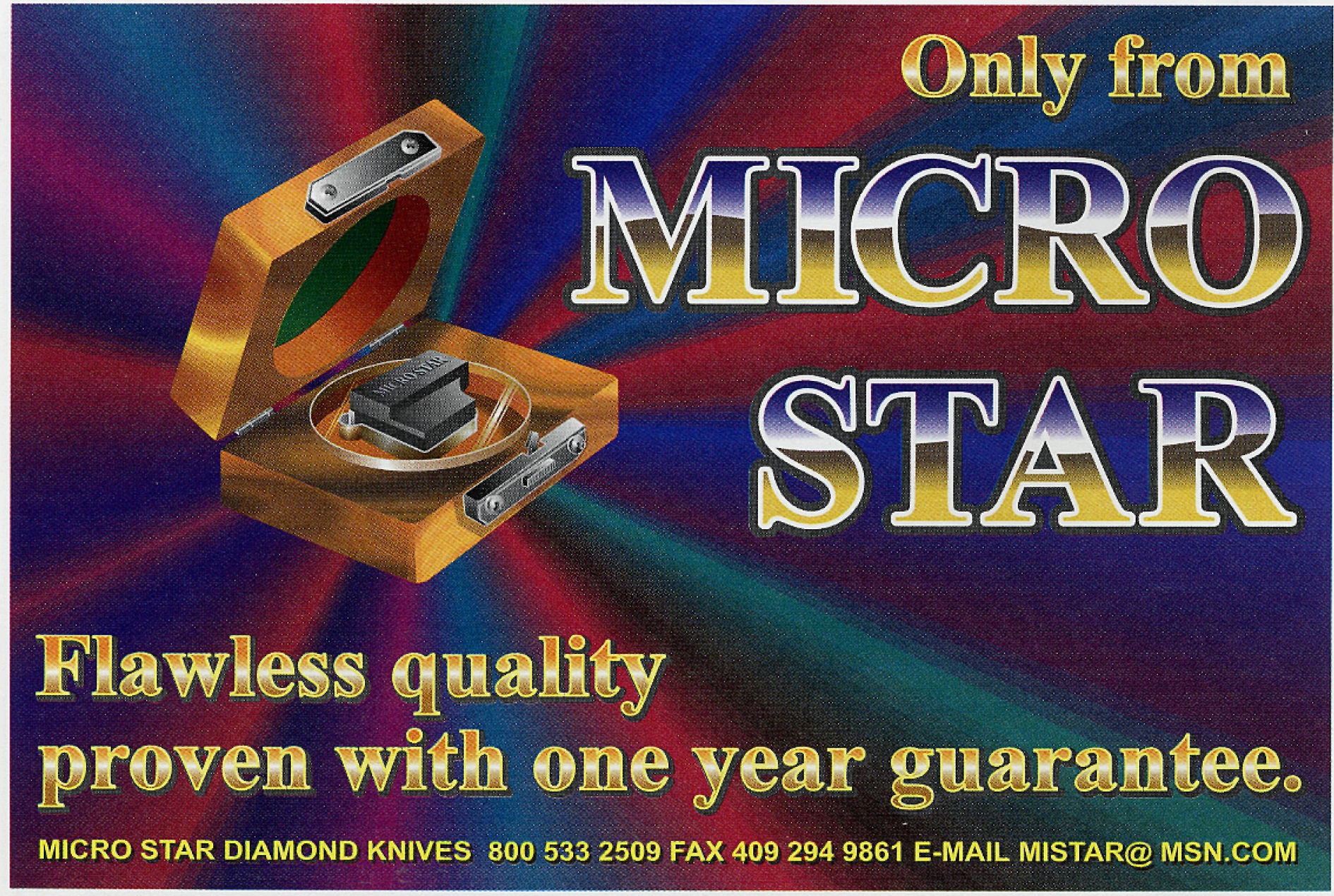




\section{The Effect of Chamber Pressure on Beam Scatter}

As the chamber pressure increases, beam scatter increases. The value in the upper left corner of each model is the chamber pressure. The value in the upper right corner is the effective beam diameter in microns. A value of 0 indicates essentially no beam scatter.

At pressures below about .1 Torr very little beam scatter occurs. But beam scatter increases very rapidly between .1 and 1 Torr and is much larger than a typical analysis area. At 10 Torr the effective beam diameter is measured in millimeters.

All four models were run in air at $15 \mathrm{~mm}$ working distance with an accelerating voltage of $20 \mathrm{kV}$.

\section{The Effect of Working Distance on Beam Scatter}

As the working distance gets longer, beam scatter increases. The value in the upper left corner of each model is the working distance. The value in the upper right corner is the effective beam diameter in microns.

The working distance for performing $X$-ray analysis is typically fixed which leaves the three other variables (pressure, $\mathrm{kV}$, and gas) to be adjusted to minimize beam scatter.

All four models were run in air at 1 TORR chamber pressure with an accelerating voltage of $20 \mathrm{kV}$.

\section{The Effect of Accelerating Voltage on Beam Scatter}

Lower accelerating voltages will result in more beam scatter. The accelerating voltage is indicated in the upper left corner of each model. The value in the upper right corner is the effective beam diameter in microns.

Accelerating voltage is inversely related to beam scatter. At higher voltages the electrons have more momentum so a scatter event will take place at a shallower angle. Increasing the accelerating voltage is an effective way to reduce the angle of scatter and minimize the skirt size.

All four models were run in a water vapor atmosphere at 1 TORR chamber pressure and $15 \mathrm{~mm}$ working distance.
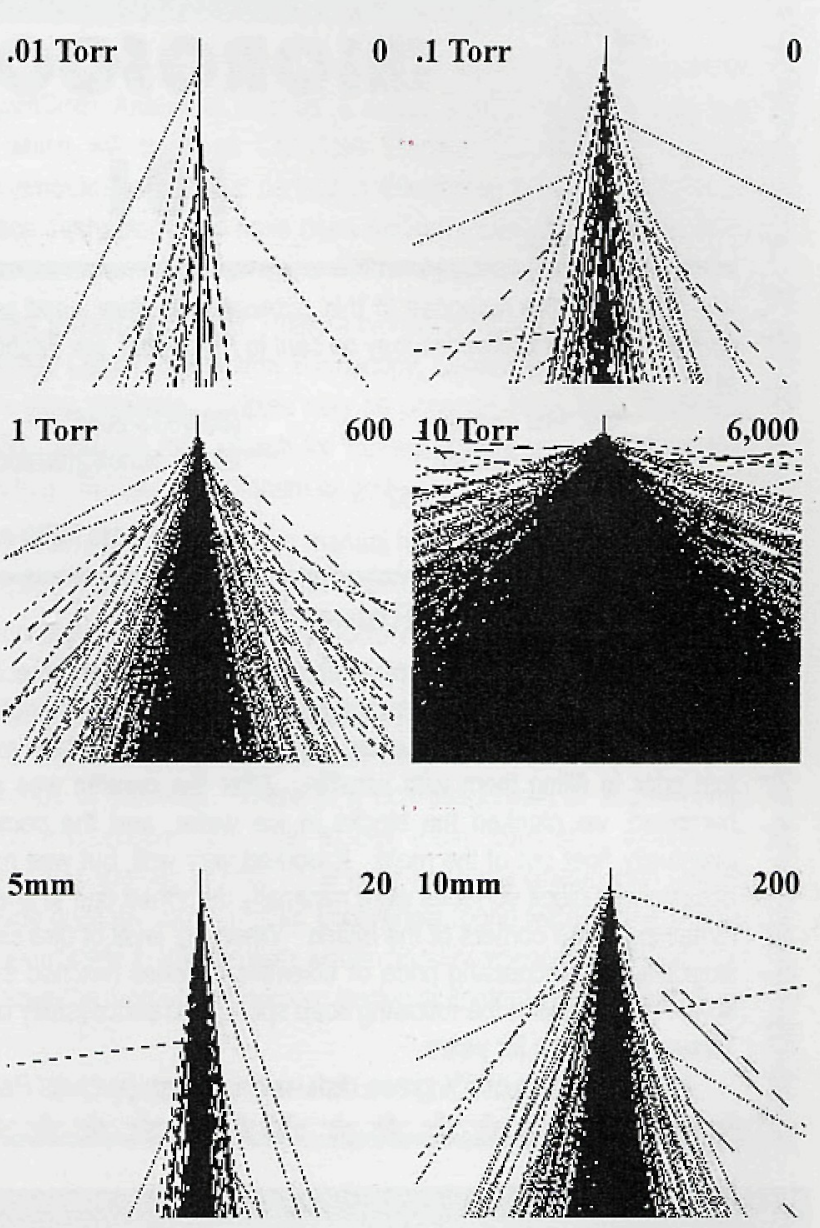

$2010 \mathrm{~mm}$
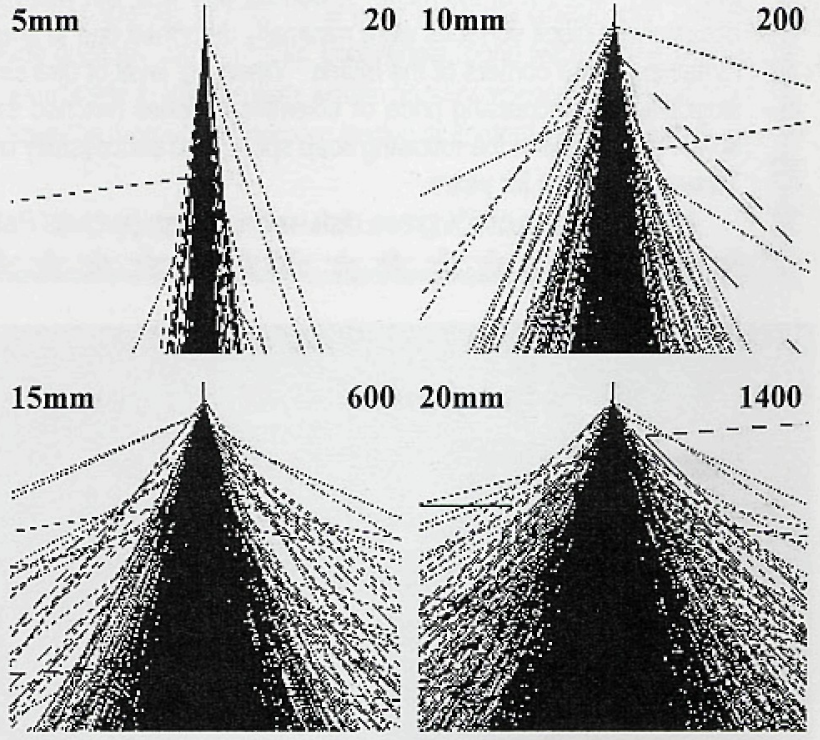

1400

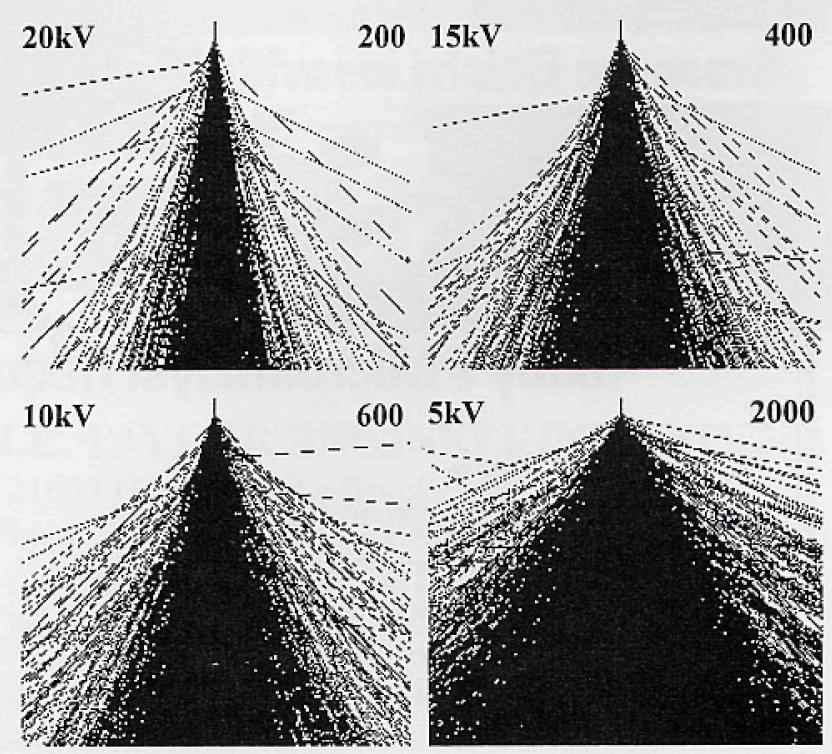

